\title{
Web-Enabled Generalized Architectural Model for Online Power System Analysis
}

\author{
${ }^{1}$ Boopathi C. Sengodan, ${ }^{1}$ Subhransu Sekhar Dash, \\ ${ }^{1}$ Subramani Chinnamuthu, ${ }^{2}$ Ramadoss Ramesh and ${ }^{3}$ Sudakaran Mahalingam \\ ${ }^{1}$ Department of Electrical and Electronics Engineering, SRM University, Chennai, India \\ ${ }^{2}$ Department of Electrical and Electronics Engineering, Anna University, Chennai, India \\ ${ }^{3}$ Department of Electrical and Electronics Engineering, Pondicherry Engineering College, Pondicherry, India
}

Received 2013-07-09, Revised 2013-07-27; Accepted 2013-08-21

\begin{abstract}
In this study, an effective Web-enabled generalized architectural model is proposed. The proposed model makes the power system analysis to be completely secured, distributed, platform-independent and languageindependent. Although significant progress has been made for on-line power system analysis in distributed environment, still efficient distributed environment has not yet been exploited. The Web-enabled architectural models are emerging as basic methodologies to support the integration of different power system applications using open Internet standard. The existing RMI models for solving multi-area power system problems in distributed environment, has its limitations to use in internet-based applets. The main objective of this study is to convert existing RMI model for power system applications into Web based model for on-line monitoring of multi-area power systems in distributed environment. Test bench has been created to carry out the performance analysis of the proposed model. Using the proposed model, load flow analysis is carried out for various bus systems. The results obtained are reported.
\end{abstract}

Keywords: On-Line Power System Analysis, Remote Method Invocation, Web Service, Grid Service, Service-Oriented Architecture

\section{INTRODUCTION}

Recent advancement in distributed computing and Internet technologies has given way to carry out the online power system analysis of large interconnected multi-area power systems in distributed environment. Remote Method Invocation (RMI) technology has been used to set up the distributed environment through which the multi-area power systems can be monitored and controlled (Nithiyanathan and Ramachandran, 2004; 2013). The RMI model makes it very easy to create Web based applets that perform powerful server side operations such as accessing a databases or communicating with remote side applications. However number of RMI limitations makes its use impractical in Internet based applets.

\subsection{State of the Art}

Various power system control centres are reviewed in detail (Wu et al., 2005). The definition and implementation of a distributed Web-based architecture for the on-line power system analysis has been analyzed (Muthu et al., 2011). The basic architecture of Web-based online real time integrated information system has been analyzed (Choi et al., 2011). The power system simulation package with various analysis distributed in a clustered environment has been modeled (Salehi et al., 2011). The package is completely web based and platform independent. An experimental illustration for on-line security analysis is carried out (Salehi et al., 2011). It is reported that the analysis could be executed in lesser period of time even for large power system. A first Web Servicesbased platform for the on-line power system monitoring is

\section{Corresponding Author: Boopathi C. Sengodan, Department of Electrical and Electronics Engineering,} SRM University, Chennai, India 
implemented (Morante et al., 2005). This analysis is a complex, large-scale and computationally complex whose solution requires different distributed resources and functionalities. The platform described in the study is able to integrate services of different kinds, such as realtime data acquisition from several sources, high computational power and data storage capabilities, to perform complex computations on large scale distributed systems (Zhang et al., 2010). An Internet interface to SCADA system is proposed ( $\mathrm{Su}$ et al., 2002). A Web model based on client/server architecture is proposed (Qiu and Gooit, 2000).

Many architectural models are proposed (Das and Roy, 2013; Ponnuramu and Tamilselvan, 2012) to carry out the on-line monitoring of multi-area power systems in distributed environment. The grid computing for monitering and control is discussed (Irving et al., 2004; Taylor et al., 2006). However, the integration of advanced distributed computing with existing architectural models becomes a practical problem. It was felt that there is a need for research enhancement and development of effective Web-enabled architectures to solve the power system problems in a completely secured, distributed, platform and language-independent environment.

Hence in this study, it is proposed to implement an effective Web-enabled generalized architectural model for making the power system analysis in a completely secured, distributed platform-independent and languageindependent environment.

\subsection{Need for Analysis in Distributed Environment}

The rapid developments of the Internet and distributed computing have opened the door for feasible and costeffective solutions for solving multi-area power system problems. Today's large interconnected power systems have very long range interconnections with many legacy systems and different operators make the maintenance of power system stability a challenging task.

The computer applications used in power system analysis have undergone profound changes since last few decades. The interconnected bulk electric power systems are becoming integrated with vast networked information systems. The existing power system operations are primarily used for desktop applications with a small number of exceptions implemented in parallel processing super computers. Many of today's desktop computing applications are distributed in nature to take the advantage of the shared resources and information available in a clustered environment. Traditionally on-line power system applications are self-contained monolithic programs that have a limited access to one another's procedure and data
(Zhang et al., 2010). They are usually cumbersome to build and expensive to maintain because simple changes in the power system operation logic may cause the entire application to be rewritten, recompiled and retested.

The present conventional client-server architecture for power system analysis is complicated, memory management is difficult, source code is bulky and exception-handling mechanism is not so easy. In the conventional power system operation and control, it is assumed that the information required for the monitoring and controlling of power systems is centrally available and all computations are to be done sequentially at a single location. With respect to sequential computation, the server has to be loaded every time for each client's request and the time taken to deliver the solution is also relatively high. A good deal of literature and implementation is available pertaining to on-line power system analysis through conventional client-server architecture. In spite of this, it is needed to develop an effective architecture to make the power system analysis in a completely platform, language and location independent environment.

\subsection{RMI Model for Power System Applications}

On-line monitoring of multi-area power system requires real-time data obtained from the different power system clients. Each sub system of interconnected power system is considered as a power system clients and these power system clients are interconnected with the server. The power system client applications are running in a heterogeneous environment.

An Remote Method Invocation (RMI) based singleserver/multiple-clients architecture has been proposed (Nithiyananthan and Ramachandran, 2005) in such a way that for every specific period of time, the remote server obtains the power system data simultaneously from the neighboring power systems which are the clients registered with the remote load flow server and the load flow solutions from the server have been sent back to the respective clients. In existing RMI models, the power system client who is deployed in Java platform cannot communicate with the load flow service that was developed and deployed using some other paradigm. Each subsystem has been considered as a power system client and hence multi power system clients-single server model is implemented.

A client computer basically does the distributed power system monitoring through an applet for every specific period of time and frequently exchanges data with the server. The server does the load flow analysis 
and then distributes the results. Chronologically the server process should be started first so that it can take the initiative to set up a connection link. It then starts waiting till it receives a connection request from the client. A client can register itself with the remote object just by invoking the registration procedure on the server object, when it needs a service from it. The remote object obtains the necessary data from the registered client objects and responds back to them respectively with the results. This total process can be automated by making the server get the input data for every specific period of time. Transaction of data between clients and server takes place several times and so the possibilities of the occurrence of errors may be high. Hence it must be handled properly.

The Limitations of using RMI in Applets (Jagannadham et al., 2007) on the Internet are the following:

- $\quad$ The most popular web browsers--Netscape Navigator and Microsoft Internet explorer do not adequately support RMI. Navigator supports RMI on most platforms but does not support HTTP tunneling. The power system clients connected to a Web through the proxy server cannot use RMI applet

- $\quad$ RMI registry can experience problem when handling connections via the Internet

- The RMI registry is not as flexible as most server applications, like answering only to the client connections made to one specific hostname this means that RMI server application cannot support multiple domains on a single web server, which limits the deployment options

- Power system applications require the data exchanged between the client and server be encrypted or compressed. The RMI requires only that implement socket classes and RMI socket factory that creates specific special sockets, however overriding the default socket factory disables RMI's ability to do HTTP tunneling, thereby preventing access by proxy server users

- Deploying RMI's application requires additional network firewall configuration by default RMI object are bound to random "anonymous" server port numbers, make it impossible to predict the ports that must be available to Internet clients. The proposed Web-based model allows specifying a port number when creating a remote object

\section{WEB-BASED COMPUTING FOR POWER SYSTEM APPLICATIONS}

Web technologies enable communication between dissimilar computers over a large geographical region via Intranet or Internet (Fig. 1). These provide a general distributed computing environment so that distributed applications can be implemented in it to exploit cheap but powerful parallel virtual machines. Web-based computing permits data sharing and computing over a large system range on heterogeneous hardware and software platforms, permitting the execution of number of operations simultaneously. This technology is basically based on client-server paradigms and concurrent programming.

The advantages that a Web-based system offers over traditional legacy systems are:

- $\quad$ Reduced costs (adding new resources, training and maintenance)

- Improved access to the system through redeployment and re-orientation of existing hardware and software resources

- Anytime, anywhere secured access to users and customers

- Easy access to users over the Internet since no extra hardware or software is required to access the application

- Ease of maintenance from a Programming/Maintenance group perspective

- User-friendly interface that requires minimal training/re-training

- Ease in deployment and enhancement of functionality

The main aim of the proposed Web-.based architectural model is to provide the service orientation for different power system applications like Load flow analysis, Contingency analysis and Economic load dispatch. The power system applications have been developed as a different web services and all are encapsulated into one frame. The components in a frame are loosely coupled each other, so legacy components can be integrated with new power system applications in future.

The features of proposed architectural models are detailed as follows:

- In RMI mechanism, the client can communicate with server only if the stub is present and the availability of stub should be taken care by the developer. But in the case of web service, automatically it take care the availability of stub 


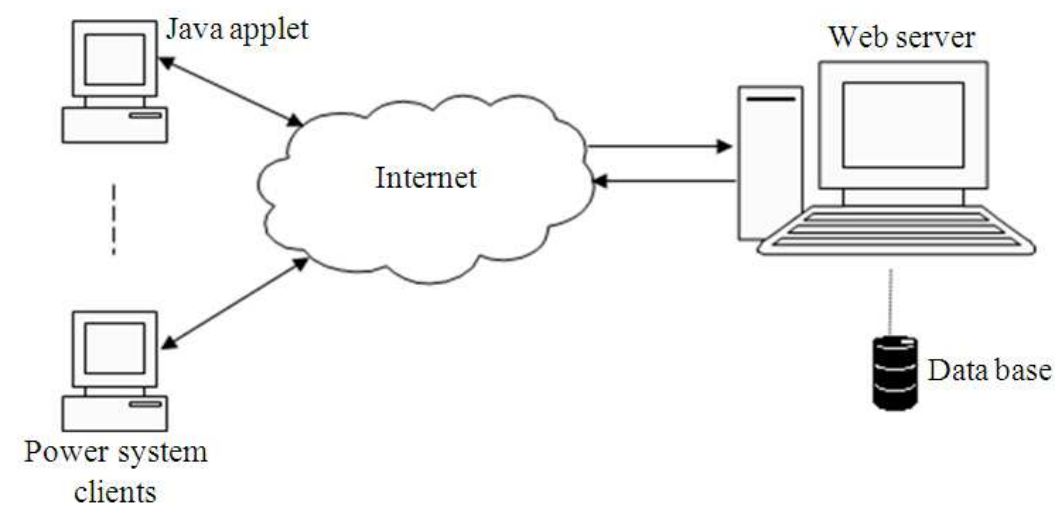

Fig. 1. Web-based share computing mode

- Java RMI use optimized connection-oriented communications protocols that are either language specific, or have detailed rules defining how datastructure and interfaces should be realized. In contrast, Web services are based on the ubiquitous technologies that have grown up to support WWWservices (human-via-browser-to-application)

- There is no concept of an object reference; instead a service is defined by an end-point that supports various operations

- Web services provide interoperability between various software applications running on disparate platforms

- Web services use open standards and protocols. Protocols and data formats are text-based where possible, making it easy for developers to comprehen

- $\quad$ By utilizing HTTP, web services can work through many common firewall security measures without requiring changes to the firewall filtering rules. Other forms of RPC may more often be blocked

- Web services allow software and services from different companies and locations to be combined easily to provide an integrated service

- Web services allow the reuse of services and components within an infrastructure

- Web services are loosely coupled thereby facilitating a distributed approach to application integration

The proposed models use the built-in security mechanism and hence the distributed on-line power system analysis through an applet definitely secures the safety of the server as well as the power system data transfer.

\section{ALGORITHM FOR IMPLEMENTATION OF ON-LINE LOAD FLOW ANALYSIS}

Both power system client and the server are considered as remote objects. The server object uses a single thread of control to distribute the load flow solutions simultaneously to the power system clients registered with it. The proposed model is dynamic which allows a new power system client to register with the load flow server object at run-time and get serviced. The servers and the clients have to store them, the necessary object codes required for calculations. Stubs for both client and server must be kept at a common location. Subsequently, the following steps are to be carried out.

- Start the load flow server

- Start the power system client by loading the server's stub from the common location

- Client registers with the server by invoking the appropriate method for the remote object

- Server uses the client's reference to receive the power system data from the associated client database

- Load flow server computes the load flow result and sends the information to the respective client

- Client obtains the result from the server through stub and provides a view of the result through an applet

- For every specific period of time, the server automatically receives power system data from the client so that the secured operation of power systems can be maintained

Any number of power system clients can be served without limit. When the server receives data from the power system client, it runs the required service 
automatically and the result is sent back to the respective power system client. Using this model, different power system clients can obtain the different services continuously at regular time intervals. Similarly the proposed algorithm can also be used to carry out the on-line contingency analysis and real-time economic load dispatch.

\section{PERFORMANCE COMPARISON AND DISCUSSION}

The above model has been implemented to carry out the on-line load flow analysis extensively for various Bus systems. The various Bus systems considered for investigation are 3, 5, 6, 9 and IEEE 30 bus power system. The sample result obtained for IEEE 30 bus system is presented in Fig. 2.

The core power system operations are being carried out in distributed environment. The distributed application has been constructed using different distributed technologies.

All the services are encapsulated in a container. The major factor that influences the performance of the proposed model is the Round Trip Time (RTT) that includes the convergence time. The round trip time measures the time needed from the point when the client sends the request and when the clients receives the solution.

The round trip time is measured for all the power system clients that invoked the load flow method simultaneously without delay. The performance analysis of the proposed grid service model has been carried out with respect to load flow monitoring and the variations of round trip time with respect to the number of clients is shown in Table 1 and Fig. 3.

Table 1. RTT Vs number of power system clients

\begin{tabular}{lllllr}
\hline & \multicolumn{5}{c}{ Execution time for 3, 5, 6, 9 and 30 in ms } \\
No. of & ----------------------- & \\
Clients & 3bus & 5 bus & 6bus & 9bus & 30bus \\
\hline 1 & 11.20 & 15.0 & 16.2 & 18.1 & 20.2 \\
2 & 20.00 & 28.0 & 28.2 & 28.8 & 31.0 \\
3 & 35.64 & 39.2 & 41.2 & 54.3 & 57.1 \\
4 & 48.90 & 55.4 & 56.3 & 58.8 & 62.0 \\
5 & 65.10 & 65.2 & 70.8 & 71.1 & 73.1 \\
6 & 85.00 & 94.0 & 98.3 & 100.1 & 101.3 \\
\hline
\end{tabular}

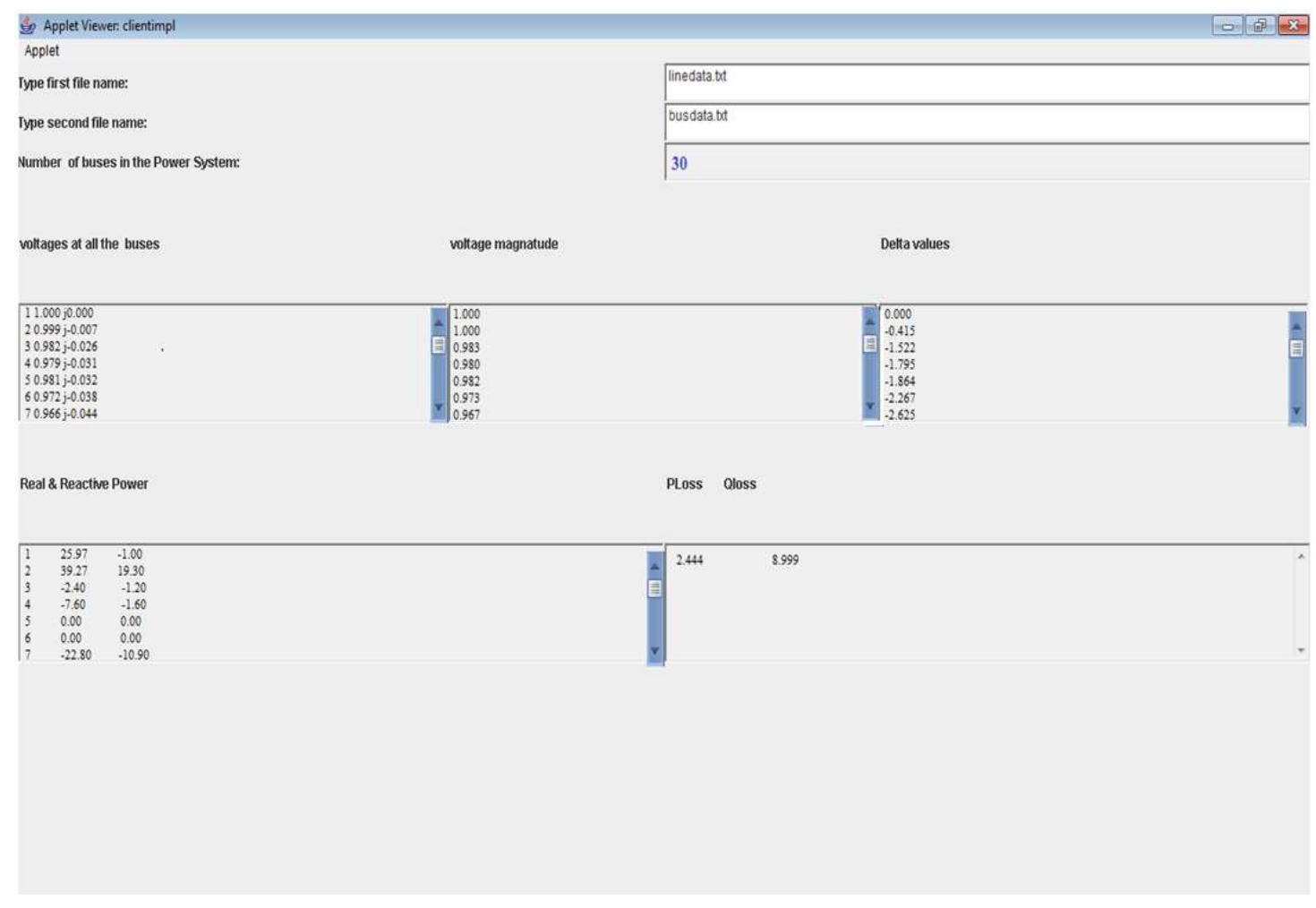

Fig. 2. Load flow solution for IEEE 30 bus system-power system client's view 


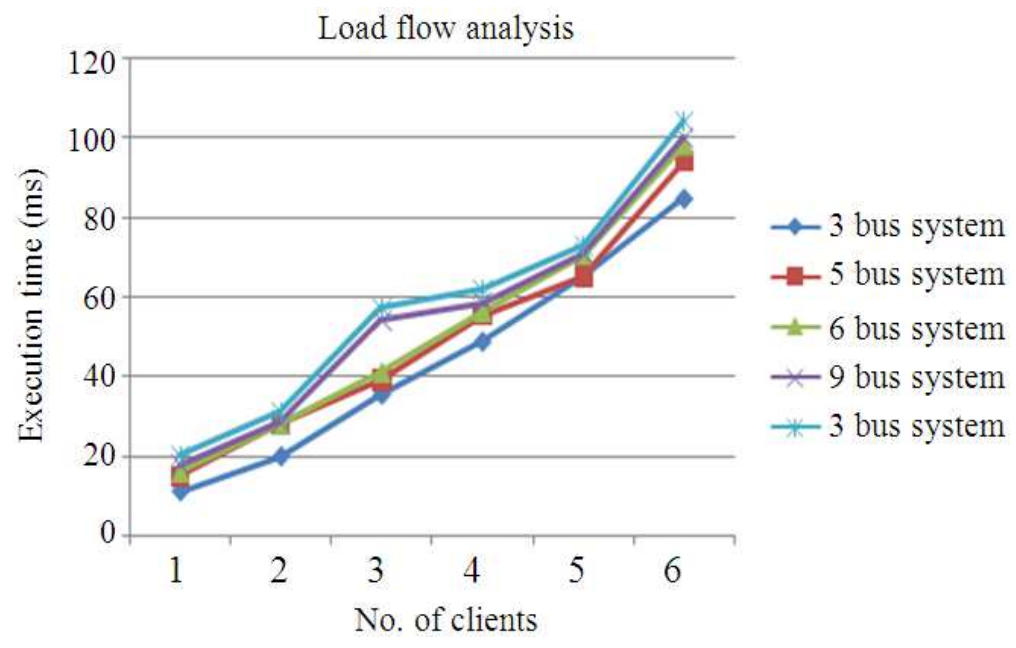

Fig. 3. RTT Vs number of power system clients

The graph is plotted between the round trip time and the number of clients registered with the server at a specific interval of time. From the graph it is found that the time taken to invoke the load flow method and to return the result increases as number of clients increases. Similarly the performance analysis of the proposed model can also be carried out for contingency analysis and economic load dispatch.

To measure the performance of different models, different distributed technologies have been used for implementation and prepared a simple test client. The test has been carried out in the following different cases:

Test 1: Server and Client on same machine (Local)

Test 2: Server and Client on Different machines (Remote Invocation)

Test 3: Server and Client on Different machines (Remote through Proxy Server Invocation)

Test 4: Different Web services invocation performance both server and client on different machines through Firewalls (Remote through Proxy Server)

Java socket is an endpoint for communication between two processes i.e., server process and client process running in the same network. RMI provides sophisticated distributed and platform-independent environment to solve the power system problems. The location transparency is the key frame of CORBA/RMIIIOP. Location transparency of the proposed model is the ability to access and invoke operations on the CORBA server object without needing to know where the power system object resides. The Web service provides platform independence, language independence and location independence (Aydogmus and Aydogmus, 2009).

Two identically configured computers have been used, one acting as client and the other server, connected in $100 \mathrm{Mbps}$ switched network. Both systems are running Microsoft Windows XP Operating Systems. Both systems have the same settings containing Pentium 4 processor with $3 \mathrm{GHz}$ speed, 1GB RAM, Java 2 Platform Standard Edition version 1.4, Java Web Services Developer Pack version 1.5, Tomcat 4.1 Server and Apache Axis.

The performance analysis was carried where all the power system services and the clients are running in the same machine. RTT has been measured. The graph is plotted between the round trip time and the different power systems, as shown in Fig. 4.

The developed power system service and the power system client are running in different machines. RTT is measured for different cases and the graph is plotted and shown in Fig. 5.

The server and the power system client are running in different machines, where the remote machine is configured with firewalls. RTT is measured for different cases and the graph is plotted and shown in Fig. 6.

The performance analysis was carried using the Web service, where the server and the clients are running in the same machine. RTT has been measured and the graph is plotted between the round trip time and the different power system, as shown in Fig. 7. 
Boopathi C. Sengodan et al. / American Journal of Applied Sciences 10 (9): 1093-1101, 2013

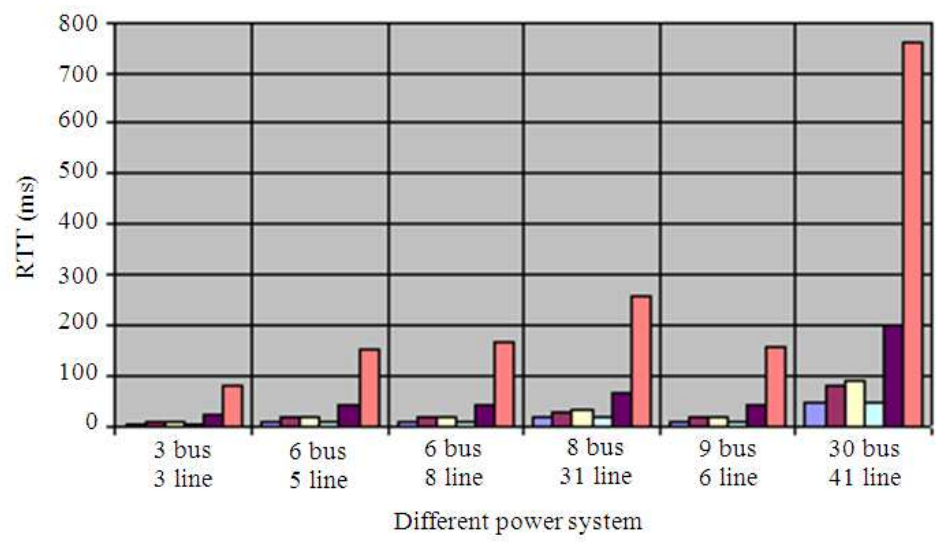

QRMI $\square$ CORBA $\square$ RMI-IIOP $\square$ SOCKET $\square$ WEB SERVICE $\square$ XML-RPC

Fig. 4. Performance analysis for test bench: Client and services in same machine

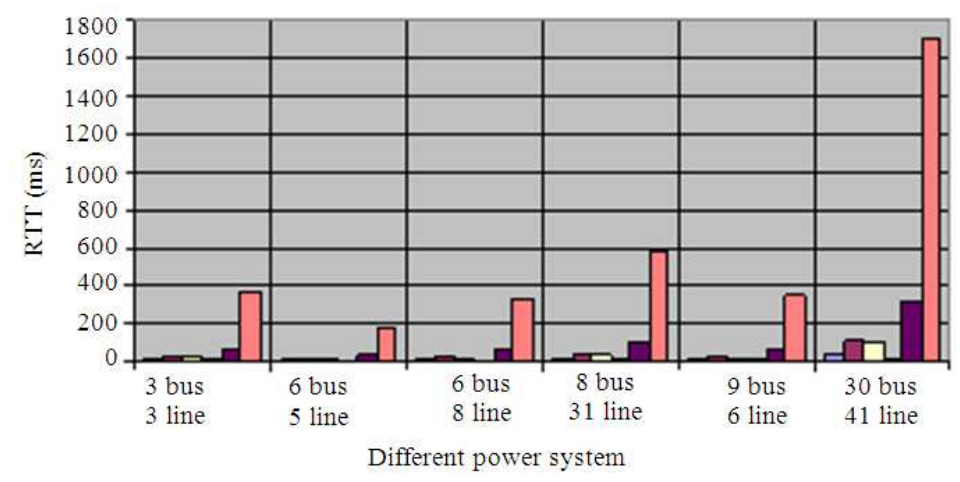

口RMI $\square$ CORBA $\square$ RMI-IIOP $\square$ SOCKET $\square$ WEB SERVICE $\square$ XML-RPC

Fig. 5. Performance analysis for test bench 2: Client and services in different machine

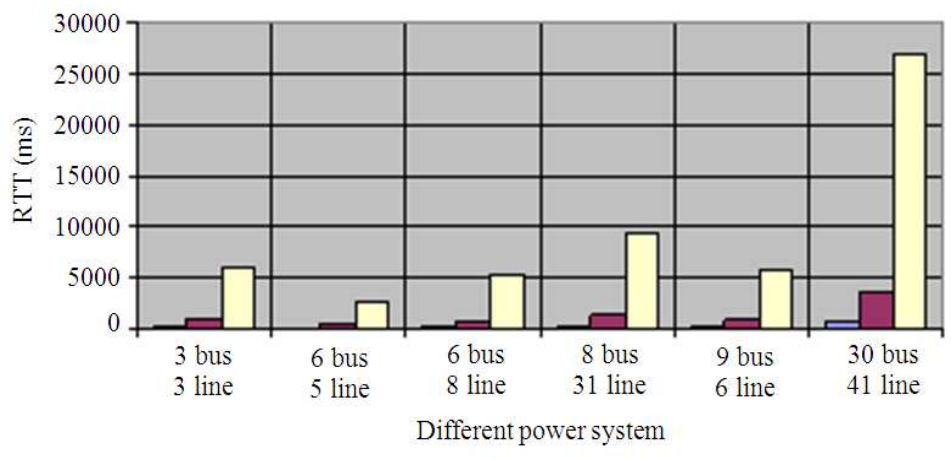

口RMI $\square$ Web service $\square$ XML-RPC

Fig. 6. Performance analysis for test bench 3: Remote 


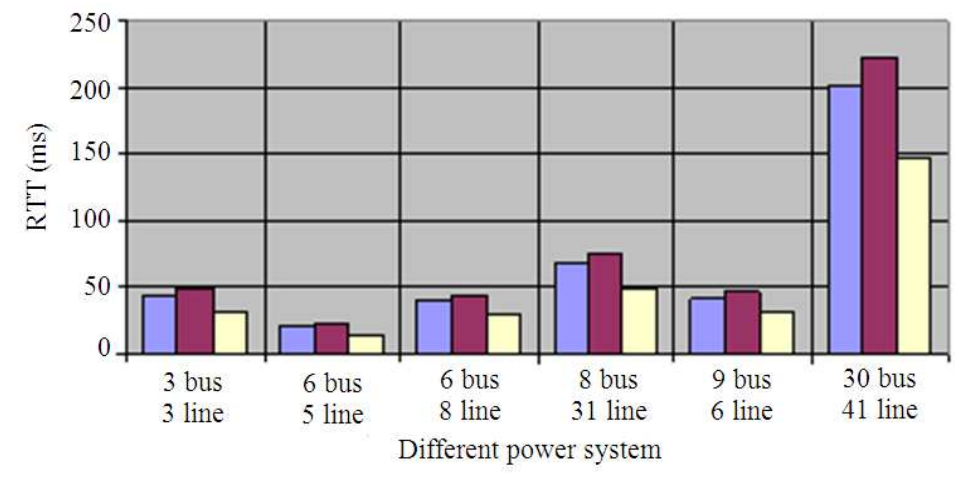

口 Stub aDII DDPROXY

Fig. 7. Performance analysis for test bench 4: Web services invocation performance

Similarly, the Web service model has been developed to carry out the on-line power system analysis and the performance analysis was carried out and RTT has been measured. It is found that the time taken to invoke the service and to run the results increase as number of clients increases. Since both SOAP and WSDL are XML-based, XML messages have to be passed on both the sides. The client side proxies have to be generated on the client side before any communication can takes place. XML parsing at runtime requires additional processing time which may result in longer response time of the server in case of a Web service server. The overhead of the Web service stems mainly from the usage of XML producing human readable text and is employed when interoperability with other Web services and applications is essential.

From the result obtained, it is observed that the RTT for Web based model is comparatively high as compared to approaches such as RMI and CORBA. But, the overhead in RTT is within the permissible limit and does not affect the system performance. The proposed Web enabled model provides a cross-platform, cross-language data model that facilitates developing heterogeneous distributed applications. XML-RPC is found a useful way to tie together the systems written in different languages on different operating systems and enabling them to co-operate. The real advantage is that the structure of XML-RPC is flexible and suitable for different power system applications.

\section{CONCLUSION}

This study presents a web based generalized model for power system analysis. Using the proposed model, load flow analysis is carried out for different bus systems. The proposed Web-based model provides the service orientation for various power system applications like load flow analysis, contingency analysis and economic load dispatch. The proposed generalized model is found to be suitable not only for power system applications, but also for private, public industries, institutions and e-governance applications.

\section{REFERENCES}

Aydogmus, Z. and O. Aydogmus, 2009. A web-based remote access laboratory using SCADA. IEEE Trans. Educ., 52: 126-132. DOI: 10.1109/TE.2008.921445

Choi, J.S., J.J. Park, T.G. Oh, K.H. Cho and J. Mitra et al., 2011. Web based online real-time information system for reliability of electrical energy supply including WTG. J. Int. Council Electr. Eng., 1: 281286. DOI: 10.5370/JICEE.2011.1.3.281

Das, H. and D.S. Roy, 2013. A Grid computing service for power system monitoring. Int. J. Comput. Applic.

Irving, M., G. Taylor and P. Hobson, 2004. Plug in to grid computing. IEEE Trans. Power Energy Mag., 2: 40-44. DOI: 10.1109/MPAE.2004.1269616

Jagannadham, D., V. Ramachandran and H.N.H. Kumar, 2007. Java2 distributed application development (Socket, RMI, Servlet, CORBA) approaches, XMLRPC and web services functional analysis and performance comparison. Proceedings of the International Symposium on Communications and Information Technologies, Oct. 17-19, IEEE Xplore Press, Sydney, NSW., pp: 1337-1342. DOI: 10.1109/ISCIT.2007.4392224. 
Morante, Q., N. Ranald and E. Zime, 2005. Web services workflow for power system security assessment. Proceedings of the IEEE International Conference on E-Technology, e-Commerce and e-Service, Mar. 29-Apr. 1, IEEE Xplore Press, pp: 374-380. DOI: 10.1109/EEE.2005.145

Muthu, B., R. Veilumuthu and L. Ponnusamy, 2011. Service oriented architectural model for load flow analysis in power systems. J. Electr. Eng., 62: 227232. DOI: $10.2478 / \mathrm{v} 10187-011-0036-9$

Nithiyananthan, V. and Ramachandran, 2005. RMI based distributed model for multi-area power system online economic load dispatch. J. Electr. Eng., 56: 4144. DOI: 10.1.1.118.5029.

Nithiyanathan, K. and V. Ramachandran, 2004. RMI based distributed database model for multi-area power system load flow monitoring. Int. J. Eng. Intell. Syst., 12: 15-19.

Nithiyanathan, K. and V. Ramachandran, 2013. Versioning-based service-oriented model for multiarea power system online economic load dispatch. Comput. Electr. Eng., 39: 433-440. DOI: 10.1016/j.compeleceng.2012.09.016

Ponnuramu, V. and L. Tamilselvan, 2012. Data integrity proof and secure computation in cloud computing. J. Comput. Sci., 8: 1987-1995. DOI: 10.3844/jcssp.2012.1987.1995

Qiu, B. and H.B. Gooit, 2000. Web-based SCADA Display Systems (WSDS) for access via Internet. IEEE Trans. Power Syst., 15: 681-686. DOI: $10.1109 / 59.867159$
Salehi, V., A. Mazloomzadeh, J.F. Fernandez and O.A. Mohammed, 2011. Real-time power system analysis and security monitoring by WAMPAC systems. Proceedings of the IEEE PES Innovative Smart Grid Technologies, Jan. 16-20, IEEE Xplore Press, Washington, DC., pp: 1-8. DOI: 10.1109/ISGT.2012.6175768

Su, C.L., C.N. Lu and T.Y. Hsiao, 2002. Simulation study of Internet based inter control center data exchange for complete network modeling. IEEE Trans. Power Syst., 17: 1177-1183. 10.1109/TPWRS.2002.805021.

Taylor, G.A., M.R. Irving, P.R. Hobson, C. Huang and P. Kyberd et al., 2006. Distributed monitoring and control of future power systems via grid computing. Proceedings of the IEEE Power Engineering Society General Meeting, (SGM' 06), IEEE Xplore Press, Montreal, Que. DOI: 10.1109/PES.2006.1709538

Wu, F.F., K. Moslehi and A. Bose, 2005. Power system control centers: Past, present and future. IEEE Proc., 9: 1890-1908. DOI: 10.1109/JPROC.2005.857499

Zhang, P., F. Li and N. Bhatt, 2010. Next-generation monitoring, analysis and control for the future smart control center. IEEE Trans. Smart Girds, 1: 186-192. DOI: $10.1109 /$ TSG.2010.2053855 